\title{
Ramsey problems in additive number theory
}

\author{
by \\ BÉla Bollobás (Cambridge), Paul Erdős (Budapest) \\ and Guoping Jin (Cambridge)
}

1. Introduction. In 1964 Erdős and Heilbronn [2] proved that if $p$ is a prime and $A$ is a set of at least $3 \sqrt{6 p}$ residues modulo $p$, then $\sum_{b \in B} b \equiv 0$ $(\bmod p)$ for a non-empty subset $B$ of $A$. Subsequently Olson [5] proved the essentially best possible result that if $A$ is a set of more than $\sqrt{4 p-3}$ non-zero residues modulo $p$ then for every integer $n$ there is a non-empty subset $B$ of $A$ such that $\sum_{b \in B} b \equiv n(\bmod p)$.

In 1985, Burr and Erdős [1] studied infinite sequences $A$ of natural numbers such that if $A=A_{1} \cup A_{2}$ then every (or every sufficiently large) number $n$ is a sum of distinct terms of some $A_{i}$, with $i$ depending on $n$. This is a Ramsey-type question (see [3], [4]) for integers: when is it true that every partition is such that at least one of the parts has a certain property?

Our aim in this note is to study some new question related to the problems above.

Denote by $f_{k}(n)$ the minimal integer $m$ such that no matter how we divide the integers from 1 to $m$ into $k$ classes, $n$ is a sum of distinct terms of one of the classes. What can one say about $f_{k}(n)$ ? Also, let us denote by $g_{k}(n)$ the minimal integer $m$ such that there is a subset $A$ of $\{1,2, \ldots, n-1\}$ with $g_{k}(n)=\sum_{a \in A} a$ such that if the integers in $A$ are partitioned into $k$ classes, then $n$ is always a sum of some integers from the same class. What can one say about $g_{k}(n)$ ? In this paper we shall investigate these two questions for $k=2$.

It is easily seen that if $\sum_{i=1}^{m} i \leq 2 n-2$ then $f_{2}(n)>m$; thus $f_{2}(n) \geq$ $\lfloor 2 \sqrt{n-1}+1 / 2\rfloor$. In Theorem 4 in the second section we shall show that this trivial lower bound is close to the true value of $f_{2}(n)$, namely that $f_{2}(n) \leq 2 \sqrt{n}+c_{0} \log n$ for some constant $c_{0}$. It is rather surprising that it seems to be difficult to improve substantially the trivial lower bound above: all we shall show is that $f_{2}(n) \geq\lfloor 2 \sqrt{n}\rfloor+2$ if $n$ is large enough (Theorem 5).

It is immediate from the definitions that $g_{k}(n) \leq f_{k}(n)\left(f_{k}(n)+1\right) / 2$ so $g_{2}(n) \leq 2 n+c_{1} \sqrt{n} \log n$. However, concerning a lower bound on $g_{2}(n)$, it is 
not even obvious that $g_{2}(n) \geq 2 n$. In Theorem 7 in the third section we shall show that $g_{2}(n)$ is substantially larger than $2 n$, in fact, $g_{2}(n) \geq 2 n+\sqrt{2 n} / 8$ if $n$ is sufficiently large.

2. Bounds for the function $f_{2}(n)$. In order to give an upper bound for $f_{2}(n)$ we need three easy lemmas. As usual, denote by $[b]$ and $[a, b]$ the sets of integers $\{i: 1 \leq i \leq b\}$ and $\{i: a \leq i \leq b\}$ respectively.

Lemma 1. Let $c<d$ and $m$ be positive integers. Suppose that $A \subset[m]$ does not contain elements $a$ and $b$ with $c \leq b-a \leq d$. Then

$$
\sigma(A) \leq \frac{c}{2(c+d)}(m+d+r+1)(m-r)+\frac{1}{2}(2 r-e+1) e,
$$

where $r=m-\lfloor m /(c+d)\rfloor(c+d)$ and $e=\min \{r, c\}$. In particular,

$$
\sigma(A) \leq \frac{c}{2(c+d)}(m+1)(m+d) .
$$

For the sake of convenience, given a finite set $A \subset \mathbb{N}$ denote by $\sigma(A)$ the total sum of its elements, and let $\Sigma(A)=\{\sigma(B): B \subset A\}$ be the set of integers that can be written as a sum of some elements of $A$. Our upper bound on $f_{2}(n)$ will be the function

$$
m(n)=\left\lfloor 2 \sqrt{n}+\log _{5 / 4} n+8\right\rfloor .
$$

LEMma 2. If $m \geq 3$ and $A \subset[m]$ contains all odd numbers not greater than $m$ then $\Sigma(A) \supset[3, \sigma(A)-3]$. In particular, if $n \geq 2$ and $A \subset[m(n)]$ contains all odd numbers not greater than $m$ then $n \in \Sigma(A)$.

Pro of. The assertion is easily checked for $3 \leq m \leq 7$; the rest follows by induction on $m$ since $\sigma(A)-5 \geq m+1$ for $m \geq 7$.

LEMma 3. For $n \geq 4$, if $[m(n)]=A_{1} \cup A_{2}$ and neither $A_{1}$ nor $A_{2}$ is the set of all odd numbers in $[m(n)]$ then for some $i$ we have

$$
\sigma\left(A_{i}\right) \geq \frac{m(n)(m(n)+1)}{4}-\frac{m(n)-3}{4}=\frac{(m(n))^{2}-3}{4}
$$

and in $A_{i}$ there are two integers with difference 1.

Proof. The lemma is trivial if both $A_{1}$ and $A_{2}$ contain two integers with difference 1. Suppose that $A_{2}$ does not contain two integers with difference 1. Then $A_{1}$ must contain two integers with difference 1 . Indeed, otherwise, one of $A_{1}$ and $A_{2}$ must be the set of all odd numbers in $[m(n)]$.

Since $A_{2}$ does not contain two integers with difference 1 , we have

$$
\begin{aligned}
\sigma\left(A_{2}\right) & \leq m(n)+(m(n)-2)+(m(n)-4)+\ldots \\
& \leq \frac{m(n)(m(n)+1)}{4}+\frac{m(n)-3}{4} .
\end{aligned}
$$


As $A_{1} \cup A_{2}=[m(n)]$, this gives

$$
\sigma\left(A_{1}\right) \geq \frac{m(n)(m(n)+1)}{4}-\frac{m(n)-3}{4}=\frac{(m(n))^{2}-3}{4},
$$

completing the proof of the lemma.

THEOREM 4. If $n$ is sufficiently large then

$$
f_{2}(n) \leq m(n)=\left\lfloor 2 \sqrt{n}+\log _{5 / 4} n+8\right\rfloor .
$$
that

Proof. Let $[m(n)]=A_{1} \cup A_{2}$. To prove the theorem, we have to show

$$
n \in \Sigma\left(A_{1}\right) \cup \Sigma\left(A_{2}\right) .
$$

By Lemmas 2 and 3 , we may assume that

$$
\sigma\left(A_{1}\right) \geq \frac{m(n)(m(n)+1)}{4}-\frac{m(n)-3}{4}=\frac{(m(n))^{2}-3}{4},
$$

and there are $a$ and $b$ in $A_{1}$ such that $b-a=1$.

We claim that $A_{1}$ contains a set $F_{l}=\left\{a_{1}, b_{1}, \ldots, a_{l}, b_{l}\right\}$, where $l \leq$ $\left\lceil\frac{1}{2} \log _{5 / 4} n\right\rceil+6$, such that the set

$$
\left\{\sum_{i=1}^{l} c_{i}: c_{i}=a_{i} \text { or } b_{i}\right\}
$$

contains the interval $[a, b]$, where $a=\sum_{i=1}^{l} a_{i}$ and $b=\sum_{i=1}^{l} b_{i}$, and this interval has length at least $m(n)$. Having proved the claim it is easy to see that $n \in \Sigma\left(A_{1}\right)$. Indeed, inequalities (1) and (2) imply that

$$
\sigma\left(A_{1}-F_{l}^{\prime}\right) \geq \frac{(m(n))^{2}-3}{4}-a>n,
$$

where $F_{l}^{\prime}=\left\{a_{1}, \ldots, a_{l}\right\}$. Let $D$ be a maximal subset of $A_{1}-F_{l}$ such that

$$
\sigma(D) \leq n-a \text {. }
$$

Then, rather crudely, $\sigma(D)+m(n) \geq n-a$, so

$$
\sigma(D)+a \leq n \leq \sigma(D)+b .
$$

Hence $n \in \Sigma\left(A_{1}\right)$, as asserted by our theorem.

Now we return to prove our claim. To construct the sequence $F_{l}$, pick elements $a_{1}, b_{1} \in A_{1}$ with $b_{1}-a_{1}=1$. Suppose we have constructed $\left\{a_{1}, b_{1}, \ldots, a_{i-1}, b_{i-1}\right\}$, where $2 \leq i \leq l$. Inequality (2) and Lemma 1 imply that there are two elements $a_{i}, b_{i} \in A_{1}-\left\{a_{1}, b_{1}, \ldots, a_{i-1}, b_{i-1}\right\}$ such that

$$
1 \leq b_{i}-a_{i} \leq \sum_{j=1}^{i-1}\left(b_{j}-a_{j}\right)+1
$$

and $b_{i}-a_{i}$ is maximal subject to (3). We get a new set $\left\{a_{1}, b_{1}, \ldots\right.$ $\left.\ldots, a_{i-1}, b_{i-1}, a_{i}, b_{i}\right\}$. This completes the construction of the set $F_{l}$. 
To see the first property of the set $F_{l}=\left\{a_{1}, b_{1}, \ldots, a_{i}, b_{i}\right\}$, note that if the integers $1 \leq c_{1} \leq c_{2} \leq \ldots \leq c_{k}$ are such that $c_{i} \leq \sum_{j=1}^{i-1} c_{j}+1$ for every $i, i=1, \ldots, k$, then every integer $m \leq \sum_{i=1}^{k} c_{i}$ can be represented as $m=\sum_{i \in I} c_{i}$ for some $I \subset[k]$. Therefore, the first property holds.

Now we shall prove that the set $F_{l}$ satisfies the second property. Lemma 1 and maximality of $b_{i}-a_{i}$ imply that

$$
\frac{1}{4} \sum_{j=1}^{i-1}\left(b_{j}-a_{j}\right) \leq b_{i}-a_{i} \leq \sum_{j=1}^{i-1}\left(b_{j}-a_{j}\right)+1
$$

for every $i=1,2, \ldots, l$. This gives that

$$
b-a=\sum_{j=1}^{l}\left(b_{j}-a_{j}\right) \geq\left(\frac{5}{4}\right)^{l-1} \geq m(n),
$$

completing the proof of the claim and so that of the theorem.

As we remarked in the introduction, the above upper bound on $f_{2}(n)$ is close to being best possible. Indeed, if $m$ is the maximal integer such that $\sum_{i=1}^{m} i \leq 2 n-2$ then $[m]$ has a subset $A_{1}=\{m, m-1, \ldots, m-j\} \cup\{m-h\}$ with $\sigma\left(A_{1}\right)=n-1$, so with $A_{2}=[m]-A_{1}$ we have $[m]=A_{1} \cup A_{2}$, $\sigma\left(A_{1}\right)=n-1$ and $\sigma\left(A_{2}\right) \leq n-1$. Hence $f_{2}(n) \geq\lfloor 2 \sqrt{n-1}+1 / 2\rfloor$. It does not seem unreasonable to conjecture that if $\sum_{i=1}^{m} i \geq 2 n$ then $f_{2}(n) \leq m+1$. Our next aim is to show that this is not the case.

THEOREM 5. If $n$ is sufficiently large then

$$
f_{2}(n) \geq\lfloor 2 \sqrt{n}\rfloor+2 .
$$

Proof. Suppose that $n \geq 1100$ and $f_{2}(n) \leq m$, where

$$
m=\lfloor 2 \sqrt{n}\rfloor+1 .
$$

Then for all partitions $[m]=A_{1} \cup A_{2}$, either $n \in \Sigma\left(A_{1}\right)$ or $n \in \Sigma\left(A_{2}\right)$. Note first that

$$
\sum_{i=1}^{m} i=m(m+1) / 2 \leq 2 n+3 \sqrt{n}+1 .
$$

Let $k$ be the integer such that

$$
l-k<n \quad \text { and } \quad l \geq n
$$

where $l=\sum_{i=k}^{m} i$. Then $k \leq \sqrt{2 n}+2$, so $m-k \geq 3$ and $l<n+k<$ $n+3 m-3 k+3$. Let $A_{1}=[k+1, m]$ and $A_{2}=[k]$. Then $n \notin \Sigma\left(A_{1}\right)$ so $n \in \Sigma\left(A_{2}\right)$. Thus $k(k+1) / 2 \geq n$. Therefore

$$
k>\sqrt{2 n}-1 \text {. }
$$


To arrive at a contradiction, we shall partition $[\mathrm{m}]$ into classes $A_{1}$ and $A_{2}$ such that $n \notin \Sigma\left(A_{i}\right)$ for any $i$. Depending on the value of $l, n \leq l<$ $n+3 m-3 k+3$, we partition the integers from 1 to $m$ into two classes $A_{1}$ and $A_{2}$ in the following way.

(i) If $n \leq l<n+m-k+1$ then let $A_{1}=[k-4, m]-\{a\}$ and $A_{2}=[k-5] \cup\{a\}$, where $a=l+k-n$.

(ii) If $n+m-k+1 \leq l<n+2 m-2 k+2$ then let $A_{1}=[k-5, m]-\{a, b\}$ and $A_{2}=[k-6] \cup\{a, b\}$, where $a, b \in[k-1, m], a \neq b$ and $l+2 k-2-n=a+b$.

(iii) If $n+2 m-2 k+2 \leq l<n+3 m-3 k+3$ then let $A_{1}=[k-6, m]-$ $\{a, b, c\}$ and $A_{2}=[k-7] \cup\{a, b, c\}$, where $a, b, c \in[k, m], a \neq b \neq c \neq a$ and $l+3 k-5-n=a+b+c$.

To complete the proof, we simply check that $n \notin \Sigma\left(A_{1}\right) \cup \Sigma\left(A_{2}\right)$ for the partitions above.

Case (i). By (6), we have

(7) $\sigma\left(A_{1}\right)=l-a+k-1+k-2+k-3+k-4=n+3 k-10>n+3 \sqrt{2 n}-13$.

Then, by (5), we have $\sigma\left(A_{2}\right)<n$, so $n \notin \Sigma\left(A_{2}\right)$.

Suppose that $n \in \Sigma\left(A_{1}\right)$. Let $Q$ be a subset of $A_{1}$ such that $n=\sigma(Q)$. Since, by (7), $\sigma\left(A_{1}\right)=n+3 k-10$ and the minimal integer of $A_{1}$ is $k-4$, we have $\left|A_{1}-Q\right| \leq 2$. But by (4) and (7), $\sigma\left(A_{1}\right)>n+3 \sqrt{2 n}-13>$ $n+m+(m-1)$ so we have $\left|A_{1}-Q\right| \geq 3$, contradicting the inequality above.

Cas e (ii). By (6), we have

$$
\begin{aligned}
\sigma\left(A_{1}\right) & =l+k-1+k-2+k-3+k-4+k-5-a-b \\
& =n+3 k-13>n+3 \sqrt{2 n}-16 .
\end{aligned}
$$

Then, by (5), we have $\sigma\left(A_{2}\right)<n$, so $n \notin \Sigma\left(A_{2}\right)$.

Suppose that $n \in \Sigma\left(A_{1}\right)$. Let $Q$ be a subset of $A_{1}$ such that $n=\sigma(Q)$. Since, by (8), $\sigma\left(A_{1}\right)=n+3 k-13$ and the minimal integer of $A_{1}$ is $k-5$, we have $\left|A_{1}-Q\right| \leq 2$. But by (4) and (8), $\sigma\left(A_{1}\right)>n+3 \sqrt{2 n}-16>$ $n+m+(m-1)$ so we have $\left|A_{1}-Q\right| \geq 3$, which is a contradiction.

Cas e (iii). By (6), we have

$$
\begin{aligned}
\sigma\left(A_{1}\right) & =l+k-1+k-2+k-3+k-4+k-5+k-6-a-b-c \\
& =n+3 k-16>n+3 \sqrt{2 n}-19 .
\end{aligned}
$$

Then, by (5), we have $\sigma\left(A_{2}\right)<n$, so $n \notin \Sigma\left(A_{2}\right)$.

Suppose that $n \in \Sigma\left(A_{1}\right)$. Let $Q$ be a subset of $A_{1}$ such that $n=\sigma(Q)$. Since, by (9), $\sigma\left(A_{1}\right)=n+3 k-16$ and the minimal integer of $A_{1}$ is $k-6$, we have $\left|A_{1}-Q\right| \leq 2$. But by (4) and (9), $\sigma\left(A_{1}\right)>n+3 \sqrt{2 n}-19>$ $n+m+(m-1)$ so we have the contradiction that $\left|A_{1}-Q\right| \geq 3$. 
It would be of interest to decide whether $f_{2}(n)-2 \sqrt{n}$ is bounded or not. We are inclined to hazard the guess that it tends to infinity.

3. Bounds for $g_{2}(n)$. As Theorem 4 implies easily that $g_{2}(n)$ is close to $2 n$, the real question is the order of $g_{2}(n)-2 n$. Since, trivially, $g_{2}(n) \leq$ $f_{2}(n)\left(f_{2}(n)+1\right) / 2$, Theorem 4 gives the following upper bound on $g_{2}(n)-2 n$.

THEOREM 6. If $n$ is sufficiently large then

$$
g_{2}(n)-2 n \leq 3 \sqrt{n} \log _{5 / 4} n .
$$

When trying to prove a good lower bound for $g_{2}(n)-2 n$, we encounter considerably more serious difficulties than in giving a lower bound for $f_{2}(n)$. It is intuitively obvious that $g_{2}(n)-2 n \geq 0$ but, somewhat surprisingly, this does not seem to be trivial to prove. Nevertheless, we shall show that the bound $5 \sqrt{n} \log _{2} n$ in Theorem 6 is not far from the truth.

THEOREM 7. If $n \geq 3$ then

$$
g_{2}(n)-2 n \geq \sqrt{2 n} / 8 .
$$

Proof. As the proof is rather long, we shall put most of the work into five lemmas. Suppose that, contrary to the assertion, there is a set $A \subset[n-1]$ such that $\sigma(A)<2 n+\sqrt{2 n} / 8$ and $n \in \Sigma\left(A_{1}\right) \cup \Sigma\left(A_{2}\right)$ for all partitions $A=A_{1} \cup A_{2}$. Let $A=\left\{a_{1}, a_{2}, \ldots, a_{m}\right\}$, where $n>a_{1}>a_{2}>$ $\ldots>a_{m}>0$.

Our first aim is to define an increasing sequence of indices $k_{0}, k_{1}, \ldots, k_{t}$. In order to make this definition somewhat more convenient, let us add an auxiliary term to the sequence $\left(a_{i}\right)_{i=1}^{m}$, namely the term $a_{m+1}=n$. Let $k_{0}$ be the minimal index such that $s\left(k_{0}\right)=\sum_{i=1}^{k_{0}} a_{i} \geq n$. Clearly $k_{0} \geq 2$. If $s\left(k_{0}\right)>n$ then set $t=0$, otherwise let $k_{1}$ be the minimal index such that

$$
s\left(k_{1}\right)=\sum_{i=1}^{k_{1}} a_{i}-a_{k_{0}} \geq n .
$$

If $s\left(k_{1}\right)>n$ then set $t=1$, otherwise let $k_{2}$ be the minimal index such that

$$
s\left(k_{2}\right)=\sum_{i=1}^{k_{2}} a_{i}-a_{k_{0}}-a_{k_{1}} \geq n .
$$

As we have the auxiliary term $a_{m+1}=n$, continuing in this way we arrive at a sequence $k_{0}, k_{1}, \ldots, k_{t}$, where $k_{j}$ is the minimal index such that $s\left(k_{j}\right)=$ $\sum_{i=1}^{k_{j}} a_{i}-\sum_{l=0}^{j-1} a_{k_{l}} \geq n$. Thus $s\left(k_{j}\right)=n$ for $j=0,1, \ldots, t-1$, and $s\left(k_{t}\right)>n$.

Let us start with some easy observations concerning the sequence $a_{k_{0}}$, $a_{k_{1}}, \ldots, a_{k_{t}}$. As $s\left(k_{0}\right)=\sum_{i=0}^{k_{0}} a_{i}=n$, we have $k_{0} \geq 2$ and

(10) $\quad a_{k_{0}}<n / k_{0} \leq n / 2$. 
Furthermore, as

$$
a_{k_{j}}=\sum_{i=k_{j}+1}^{k_{j+1}} a_{i}
$$

holds for $j=0,1, \ldots, t-2$, we have

$$
a_{k_{j+1}}<a_{k_{j}} /\left(k_{j+1}-k_{j}\right) \leq a_{k_{j}} / 2,
$$

implying

$$
\sum_{j=0}^{t-1} a_{k_{j}}<a_{k_{0}} \sum_{j=0}^{t-1} 2^{-j}<2 a_{k_{0}}<n .
$$

Our next aim is to prove a simple lemma claiming that, in fact, the auxiliary term $a_{m+1}$ is not needed in the definition above.

LEMMA 8. In the notation above, we have $k_{t} \leq m$.

Pro of. Suppose that, contrary to the assertion, $k_{t}=m+1$. Then $t \geq 1$ and so

$$
s\left(k_{t-1}\right)=\sum_{i=1}^{k_{t-1}} a_{i}-\sum_{j=0}^{t-2} a_{k_{j}}=n
$$

and

$$
a_{k_{t-1}}>a_{k_{t-1}+1}+a_{k_{t-1}+2}+\ldots+a_{m} .
$$

Consequently,

$$
\sum_{i=1}^{m} a_{i}-\sum_{j=0}^{t-1} a_{k_{j}}<n .
$$

Inequalities (12) and (13) suggest a partition $A=A_{1} \cup A_{2}$ contradicting our assumption on $A$ : setting $A_{1}=\left\{a_{k_{j}}: 0 \leq j<t\right\}$ and $A_{2}=A-A_{1}$, clearly $n \notin \Sigma\left(A_{1}\right) \cup \Sigma\left(A_{2}\right)$.

Our next lemma is a considerable extension of Lemma 8: not only do we have $k_{t} \leq m$ but also the sum $a_{k_{t}+1}+a_{k_{t}+2}+\ldots+a_{m}$ is quite large. In the proof of this lemma, and in the rest of the proof of Theorem 7, we shall make use of two sets, namely

$$
K=\left\{a_{k_{0}}, a_{k_{1}}, \ldots, a_{k_{t-1}}\right\}, \quad L=\left\{a_{1}, a_{2}, \ldots, a_{k_{t}}\right\}-K .
$$

Note that $\sigma(L)=s\left(k_{t}\right)>n$ and $\sigma(K)<\sigma(L)-a_{k_{t}}<n$.

Lemma 9. We have $a_{k_{t}} \geq \sqrt{2 n} / 4$ and

$$
\sum_{i=k_{t}+1}^{m} a_{i} \geq n / 16 \text {. }
$$


Pr o of. Clearly, inequality (14) implies that $a_{k_{t}} \geq a_{k_{t}+1}+1 \geq \sqrt{2 n} / 4$, so it suffices to prove (14).

First we assume that $t \leq 3$. Let $A_{1}=L$ and $A_{2}=A-L=K \cup$ $\left\{a_{k_{t}+1}, \ldots, a_{m}\right\}$. By the definition of $k_{i}$, we have $n \notin \Sigma\left(A_{1}\right)$ so, by our assumption, $n$ must be in the set $\Sigma\left(A_{2}\right)$. A fortiori,

$$
\sigma\left(A_{2}\right)=\sum_{i=0}^{t-1} a_{k_{i}}+\sum_{i=k_{t}+1}^{m} a_{i} \geq n,
$$

so, recalling (10) and (11), we find that

$$
\sum_{i=k_{t}+1}^{m} a_{i} \geq n-\sum_{i=0}^{t-1} a_{k_{i}}>n-a_{k_{0}} \sum_{i=0}^{t-1} 2^{-i}>n-\frac{n}{2} \sum_{i=0}^{2} 2^{-i}=n / 8 .
$$

Assume now that $t \geq 4$ and (14) is false. We claim that $k_{0}=2, k_{1}=4$ and $k_{2}=6$. Indeed, if $k_{0} \geq 3$ then with $A_{1}=L$ and $A_{2}=A-A_{1}=$ $K \cup\left\{a_{k_{t}+1}, \ldots, a_{m}\right\}$ we have $n \notin \Sigma\left(A_{1}\right)$ so $n \in \Sigma\left(A_{2}\right)$. Consequently, analogously to (15), we have

$$
\sum_{i=k_{t}+1}^{m} a_{i}>n-a_{k_{0}} \sum_{i=0}^{t-1} 2^{-i}>n-\frac{n}{k_{0}} \sum_{i=0}^{2} 2^{-i}>n-2 n / k_{0} \geq n / 3 .
$$

This shows that, contrary to our assumption, (14) does hold. The assertions $k_{1}=4$ and $k_{2}=6$ are proved in a similar manner, by making use of the inequality in (11) for $j=0$ and $j=1$.

As $n=a_{1}+a_{2}, a_{2}=a_{3}+a_{4}$ and $a_{4}=a_{5}+a_{6}$, we have $a_{2}<n / 2$, $a_{4}<n / 4$ and $a_{6}<n / 8$; furthermore,

$$
a_{1}+a_{3}+a_{5}+a_{6}=a_{1}+a_{3}+a_{4}=a_{1}+a_{2}=n
$$

and

$$
a_{2}+a_{3}+a_{5}+a_{6}=a_{2}+a_{3}+a_{4}=2 a_{2}<a_{1}+a_{2}=n .
$$

Our next aim is to show that

$$
a_{1}-a_{2}+a_{3}-a_{4}+a_{5}-a_{6}>n / 16 .
$$

To this end, let $A_{1}=\left\{a_{2}, a_{3}, a_{4}, a_{5}, a_{6}\right\}$ and $A_{2}=\left\{a_{1}, a_{7}, a_{8}, \ldots, a_{m}\right\}$. By assumption, either $n \in \Sigma\left(A_{1}\right)$ or $n \in \Sigma\left(A_{2}\right)$.

In the first case, as $\sigma\left(A_{1}\right)-a_{4}<n$ by inequality (17), we have

$$
2 a_{2}+a_{6}=a_{2}+\left(a_{3}+a_{4}\right)+a_{6}=\sigma\left(A_{1}\right)-a_{5} \leq n .
$$

As $a_{4}+3 a_{6} / 2<7 n / 16$, inequalities (16) and (19) imply that 


$$
\begin{aligned}
a_{1}-a_{2}+a_{3}-a_{4}+ & a_{5}-a_{6} \\
& =a_{1}+a_{3}+a_{5}+a_{6}-\left(a_{2}+a_{6} / 2\right)-\left(a_{4}+3 a_{6} / 2\right) \\
& >n-n / 2-7 n / 16=n / 16 .
\end{aligned}
$$

In the second case $n \leq \sigma\left(A_{2}\right)$ so

$$
\begin{aligned}
2 a_{2}+a_{4} & =a_{2}+\left(a_{3}+a_{4}\right)+\left(a_{5}+a_{6}\right)=\sigma\left(A_{1}\right) \\
& =\sigma(A)-\sigma\left(A_{2}\right)<2 n+\sqrt{2 n} / 8-n=n+\sqrt{2 n} / 8 .
\end{aligned}
$$

As $a_{4} / 2+2 a_{6}<3 n / 8$, inequalities (16) and (20) imply that

$$
\begin{aligned}
a_{1}-a_{2}+a_{3}- & a_{4}+a_{5}-a_{6} \\
& =a_{1}+a_{3}+a_{5}+a_{6}-\left(a_{2}+a_{4} / 2\right)-\left(a_{4} / 2+2 a_{6}\right) \\
& >n-n / 2-\sqrt{2 n} / 16-3 n / 8=n / 8-\sqrt{2 n} / 16 \geq n / 16,
\end{aligned}
$$

completing the proof of (18).

Armed with (18), the proof of our lemma is easily completed. Indeed, set $A_{1}=L-\left\{a_{k_{t}}\right\}$ and $A_{2}=A-A_{1}=K \cup\left\{a_{k_{t}}, a_{k_{t}+1}, \ldots, a_{m}\right\}$. Then $\sigma\left(A_{1}\right)<n$ so $\sigma\left(A_{2}\right) \geq n$. Hence, by inequality (18),

$$
\begin{aligned}
\sum_{i=k_{t}+1}^{m} a_{i} & =\sigma\left(A_{2}\right)-\sigma(K)-a_{k_{t}} \geq n-\sum_{j=0}^{t} a_{k_{j}} \\
& >\sigma\left(A_{1}\right)-\sum_{j=0}^{t} a_{k_{j}} \geq \sum_{j=0}^{t}\left(a_{k_{j}-1}-a_{k_{j}}\right) \\
& >a_{1}-a_{2}+a_{3}-a_{4}+a_{5}-a_{6}>n / 16
\end{aligned}
$$

as claimed.

From Lemma 9 and the definitions of $k_{t}$ and $s\left(k_{t}\right)$, we easily deduce two more lemmas.

Lemma 10. Let $Q$ be a set of integers with $\sigma(Q)<n+a_{k_{t}}-s\left(k_{t}\right)$. Then $n \notin \Sigma(L \cup Q)$.

Proof. Let us assume that $n \in \Sigma(L \cup Q)$. Then there is a set $Q_{1}$ such that $Q_{1} \subset L \cup Q$ and $\sigma\left(Q_{1}\right)=n$. As $\sigma(L)>n$, there is an $a_{j}$ in $L$ such that $a_{j} \notin Q_{1}$. Therefore

$$
\begin{aligned}
\sigma\left(Q_{1}\right) & \leq \sigma(L \cup Q)-a_{j} \leq s\left(k_{t}\right)-a_{k_{t}}+\sigma(Q) \\
& <s\left(k_{t}\right)-a_{k_{t}}+n+a_{k_{t}}-s\left(k_{t}\right) \leq n .
\end{aligned}
$$

Lemma 11. $n+a_{k_{t}}-s\left(k_{t}\right)>\sqrt{2 n} / 8$.

Proof. Let $A_{1}=L$ and $A_{2}=A-L$. Then $n \notin \Sigma\left(A_{1}\right)$, so $n \in \Sigma\left(A_{2}\right)$. As $\sigma(A)<2 n+\sqrt{2 n} / 8$, we have

$$
s\left(k_{t}\right)=\sigma\left(A_{1}\right)<2 n+\sqrt{2 n} / 8-\sigma\left(A_{2}\right) \leq n+\sqrt{2 n} / 8 .
$$


Therefore, by Lemma 9,

$$
n+a_{k_{t}}-s\left(k_{t}\right)>n+\sqrt{2 n} / 4-(n+\sqrt{2 n} / 8)=\sqrt{2 n} / 8 .
$$

Before we can complete the proof of Theorem 7, we need one more lemma; this lemma is the heart of the entire proof. For the sake of convenience, let us extend the sequence $a_{1}>a_{2}>\ldots>a_{m}$ by the trivial term $a_{m+1}=0$.

LEMMA 12. There is an index $h$ with $k_{t}+1 \leq h \leq m+1$ such that $n \notin \Sigma\left(L \cup\left\{a_{h}\right\}\right)$ and

$$
\sigma(K)+\sum_{i=k_{t}+1}^{h-1} a_{i}<n .
$$

Pr o of. We shall consider two cases.

Case $1 . k_{t}-k_{t-1} \geq 3$. We shall make use of the set

$$
B=\left\{a_{j}+n-s\left(k_{t}\right): k_{t-1}+1 \leq j \leq k_{t}\right\} .
$$

First assume that there is an index $h$ with $k_{t}+1 \leq h \leq k_{t}+\left(k_{t}-k_{t-1}\right)$ such that $a_{h} \notin B$. It is easy to check that $n \notin \Sigma\left(L \cup\left\{a_{h}\right\}\right)$. Indeed, if $n \in \Sigma\left(L \cup\left\{a_{h}\right\}\right)$, i.e. there is a set $Q \subset L \cup\left\{a_{h}\right\}$ such that $n=\sigma(Q)$, then $Q=L \cup\left\{a_{h}\right\}-\left\{a_{i}\right\}$ for some $a_{i}$ in $L$, so $n=\sigma(Q)=s\left(k_{t}\right)+a_{h}-a_{i}$. Since $a_{h} \notin B$, we have $i<k_{t-1}$, so $a_{i}>a_{t-1}>a_{k_{t-1}+1}+a_{k_{t-1}+2}$. Thus

$$
\begin{aligned}
n & =s\left(k_{t}\right)+a_{h}-a_{i}<s\left(k_{t}\right)+a_{h}-a_{k_{t-1}} \\
& <s\left(k_{t}\right)+a_{h}-a_{k_{t-1}+1}-a_{k_{t-1}+2}<s\left(k_{t}\right)-a_{k_{t}}<n,
\end{aligned}
$$

which is a contradiction. Therefore $n \notin \Sigma\left(L \cup\left\{a_{h}\right\}\right)$, showing the first assertion of the lemma. To see the second assertion, note that as $j \leq$ $k_{t}+\left(k_{t}-k_{t-1}\right)$, we have

$$
\sigma(K)+\sum_{i=k_{t}+1}^{j-1} a_{i}<\sum_{i=1}^{k_{t-1}} a_{i}-\sigma(K)+\sum_{i=k_{t-1}+1}^{k_{t}-1} a_{i}=\sigma(L)-a_{k_{t}}<n .
$$

Assume now that $a_{j} \in B$ for all $j$ with $k_{t}+1 \leq j \leq k_{t}+\left(k_{t}-k_{t-1}\right)$ and so $B=\left\{a_{k_{t}+1}, a_{k_{t}+2}, \ldots, a_{2 k_{t}-k_{t-1}}\right\}$. Then, in particular, $a_{2 k_{t}-k_{t-1}}=$ $a_{k_{t}}+n-s\left(k_{t}\right)$. We shall show that $h=2 k_{t}-k_{t}+1$ will do. Clearly,

$\sigma\left(L \cup\left\{a_{h}\right\}\right)-a_{k_{t}}=\sigma\left(L \cup\left\{a_{2 k_{t}-k_{t-1}+1}\right\}\right)-a_{k_{t}}<\sigma(L)+a_{2 k_{t}-k_{t-1}}-a_{k_{t}}=n$. As $a_{k_{t}}$ is the smallest term in $L$ and $\sigma(L)>n$, this implies that $n \notin$ $\Sigma\left(L \cup\left\{a_{h}\right\}\right)$. Furthermore,

$$
\left\{a_{k_{0}-1}, a_{k_{1}-1}, \ldots, a_{k_{t-1}-1}\right\} \subset L-\left\{a_{k_{t-1}+1}, a_{k_{t-1}+2}, \ldots, a_{k_{t}}\right\},
$$


SO

$$
\sigma(K)=\sum_{i=0}^{t-1} a_{k_{i}}<\sum_{i=0}^{t-1} a_{k_{i}-1} \leq \sigma(L)-\sum_{i=k_{t-1}+1}^{k_{t}} a_{i}
$$

implying

$$
\begin{aligned}
\sigma(K)+\sum_{j=k_{t}+1}^{h-1} a_{j} & <\sigma(L)-\sum_{i=k_{t-1}+1}^{k_{t}} a_{i}+\sum_{j=k_{t}+1}^{h-1} a_{j} \\
& =\sigma(L)-\sum_{i=1}^{k_{t}-k_{t-1}}\left(a_{k_{t-1}+i}-a_{k_{t}+i}\right) \\
& \leq \sigma(L)+a_{h-1}-a_{k_{t}}=n
\end{aligned}
$$

Therefore Lemma 12 holds if $k_{t}-k_{t-1} \geq 3$.

Case 2. $k_{t}-k_{t-1}=2$. This time we set

$$
B=\left\{a_{i}+n-s\left(k_{t}\right): k_{t-2}+1 \leq i \leq k_{t}, i \neq k_{t-1}\right\}
$$

and $b=k_{t}-k_{t-2}-1=|B|$. Since $s\left(k_{t}\right)=\sum_{i=1}^{k_{t}} a_{i}-\sum_{i=0}^{t-1} a_{k_{i}}<n+\sqrt{2 n} / 8$, $n=s\left(k_{t-1}\right)=\sum_{i=1}^{k_{t-1}} a_{i}-\sum_{i=0}^{t-2} a_{k_{i}}$, and, by Lemma $9, a_{k_{t}}>\sqrt{2 n} / 4$, we have

$$
\begin{aligned}
a_{k_{t-1}} & =s\left(k_{t-1}\right)-\sum_{i=1}^{k_{t-1}-1} a_{i}+\sum_{i=0}^{t-2} a_{k_{i}} \\
& =s\left(k_{t-1}\right)-\sum_{i=1}^{k_{t-1}} a_{i}+\sum_{i=0}^{t-1} a_{k_{i}}=n-s\left(k_{t}\right)+a_{k_{t}-1}+a_{k_{t}} \\
& >a_{k_{t}-1}+a_{k_{t}}-\sqrt{2 n} / 8>a_{k_{t}-1}+a_{k_{t}} / 2 .
\end{aligned}
$$

Let us assume first that $k_{t-2}-k_{t-1} \geq 4$ so that $b \geq 5$ and $a_{k_{t-2}} \geq$ $a_{k_{t-2}+1}+a_{k_{t-2}+2}+a_{k_{t-2}+3}$. Let $h$ be the first index in the interval $k_{t}+1 \leq$ $h \leq k_{t}+b+1$ such that $a_{h} \notin B$. Then $n \notin \Sigma\left(L \cup\left\{a_{h}\right\}\right)$. Indeed, suppose that $n \in \Sigma\left(L \cup\left\{a_{h}\right\}\right)$, i.e. there is a set $Q \subset L \cup\left\{a_{h}\right\}$ with $n=\sigma(Q)$. As

$$
\sigma\left(L \cup\left\{a_{h}\right\}-\left\{a_{k_{t}}, a_{k_{t}-1}\right\}\right)=s\left(k_{t}\right)-a_{k_{t}}-a_{k_{t}-1}+a_{h}<s\left(k_{t}\right)-a_{k_{t}}<n,
$$

the set $Q$ is of the form $Q=L \cup\left\{a_{h}\right\}-\left\{a_{i}\right\}$ for some $a_{i} \in L$ so $n=\sigma(Q)=$ $s\left(k_{t}\right)+a_{h}-a_{i}$. Therefore $a_{h}=a_{i}+n-s\left(k_{t}\right)$, and as $a_{h} \notin B$, we have $i \notin\left[k_{t-2}+1, k_{t}\right]$ so $i \leq k_{t-2}$ and $a_{i} \geq a_{k_{t-2}}>a_{k_{t-2}+1}+a_{k_{t-2}+2}$. Thus

$$
\begin{aligned}
n & =s\left(k_{t}\right)+a_{h}-a_{i}<s\left(k_{t}\right)+a_{h}-a_{k_{t-2}+1}-a_{k_{t-2}+2} \\
& <s\left(k_{t}\right)-a_{k_{t-2}+1}<s\left(k_{t}\right)-a_{k_{t}}<n,
\end{aligned}
$$

which is a contradiction. Therefore $n \notin \Sigma\left(L \cup\left\{a_{h}\right\}\right)$, showing the first assertion of the lemma. 
Let us turn to the proof of (21). If $h \leq k_{t}+b-1$, we can see (21) as follows:

$$
\begin{aligned}
\sigma(K)+\sum_{i=k_{t}+1}^{h-1} a_{i} & =\sum_{i=0}^{t-1} a_{k_{i}}+\sum_{i=k_{t}+1}^{h-1} a_{i}<\sum_{i=0}^{t-1} a_{k_{i}-1}+\sum_{i=k_{t}+1}^{h-1} a_{i} \\
& \leq\left(\sigma(L)-\sum_{j=k_{t-2}+1}^{k_{t-1}-2} a_{j}-\sum_{j=k_{t-1}+1}^{k_{t}} a_{j}\right)+\sum_{i=k_{t}+1}^{h-1} a_{i} \\
& =\sigma(L)-\left(\sum_{j=k_{t-2}+1}^{k_{t-1}-2} a_{j}+\sum_{j=k_{t-1}+1}^{k_{t}-1} a_{j}-\sum_{i=k_{t}+1}^{h-1} a_{i}\right)-a_{k_{t}} \\
& <\sigma(L)-a_{k_{t}}=s\left(k_{t}\right)-a_{k_{t}}<n .
\end{aligned}
$$

Thus we may suppose that $h=k_{t}+b$ or $h=k_{t}+b+1$. Then $a_{k_{t}+1}, a_{k_{t}+2}, \ldots, a_{h-1} \in B$ so $a_{h-1}=a_{l}+n-s\left(k_{t}\right)$ for some $l$ with $l-k_{t-2} \geq$ $h-k_{t}$. Hence $l \geq h-k_{t}+k_{t-2} \geq b+k_{t-2}=k_{t}-1$. Therefore, arguing as above, by (22) we have

$$
\begin{aligned}
\sigma(K)+\sum_{i=k_{t}+1}^{h-1} a_{i}< & \sigma(L)-\sum_{j=k_{t-2}+1}^{k_{t-1}-2} a_{j}-\sum_{j=k_{t-1}+1}^{k_{t}} a_{j}+\sum_{i=k_{t}+1}^{h-1} a_{i} \\
< & s\left(k_{t}\right)-\sum_{j=k_{t-2}+1}^{k_{t-2}+2} a_{j}-\sum_{j=k_{t-2}+3}^{k_{t-1}-2} a_{j}-\sum_{j=k_{t-1}+1}^{k_{t}} a_{j} \\
& +\sum_{i=k_{t}+1}^{k_{t}+3} a_{i}+\sum_{i=k_{t}+4}^{h-2} a_{i}+a_{h-1} \\
= & s\left(k_{t}\right)-\left(\sum_{j=k_{t-2}+1}^{k_{t-2}+2} a_{j}-\sum_{i=k_{t}+1}^{k_{t}+3} a_{i}\right) \\
& -\left(\sum_{j=k_{t-2}+3}^{k_{t-1}-2} a_{j}+\sum_{j=k_{t-1}+1}^{k_{t}} a_{j}-a_{l}-\sum_{i=k_{t}+4}^{h-2} a_{i}\right) \\
& +a_{h-1}-a_{l} .
\end{aligned}
$$

The sums in the parentheses are non-negative: the first by inequality (22), and the second as it has $\left(k_{t-1}-k_{t-2}-4\right)+\left(k_{t}-k_{t-1}\right)$ positive terms and $1+\left(h-k_{t}-5\right) \leq\left(k_{t-1}-k_{t-2}-4\right)+\left(k_{t}-k_{t-1}\right)$ smaller negative terms. Thus,

$$
\sigma(K)+\sum_{i=k_{t}+1}^{h-1} a_{i}<s\left(k_{t}\right)+a_{h-1}-a_{l}=n .
$$

Hence the lemma holds if $k_{t-2}-k_{t-1} \geq 4$. 
Let us assume then that $k_{t-2}-k_{t-1} \leq 3$. Suppose first that there is an $h$ such that $k_{t}+1 \leq h \leq k_{t}+b-1$ and $a_{h} \notin B$. Then, arguing as above, we find that $n \notin \Sigma\left(L \cup\left\{a_{h}\right\}\right)$, and, as $h \leq k_{t}+b-1$,

$$
\sigma(K)+\sum_{i=k_{t}+1}^{h-1} a_{i}<s\left(k_{t}\right)-a_{t}<n,
$$

as required.

Suppose then that $a_{j} \in B$ for all $j$ with $k_{t}+1 \leq j \leq k_{t}+b-1$. Then either (i) $a_{k_{t}+b-1}=a_{k_{t}-1}+n-s\left(k_{t}\right)$ or (ii) $a_{k_{t}+b-1}=a_{k_{t}}+n-s\left(k_{t}\right)$.

To complete the proof of our lemma, we shall show that the assertions of the lemma hold in these two cases.

(i) Assume that $a_{k_{t}+b-1}=a_{k_{t}-1}+n-s\left(k_{t}\right)$. Since $a_{k_{t}+b-2}, a_{k_{t}+b-3}, \ldots$ $\ldots, a_{k_{t}+1}$ are all in $B$, they are all of the form $a_{i}+n-s\left(k_{t}\right)$, where $a_{i} \in L$. We have $a_{k_{t}+b-2} \geq a_{k_{t}-3}, a_{k_{t}+b-3} \geq a_{k_{t}-4}, \ldots$ and

$$
a_{k_{t}+1} \geq a_{k_{t-2}+1}+n-s\left(k_{t}\right) \text {. }
$$

In fact, as $d_{k_{t}+1}=a_{i}+n-s\left(k_{t}\right)$ for some $i \geq k_{t-2}+1$, we have equality in $\left(23^{\prime}\right)$ :

$$
a_{k_{t}+1}=a_{k_{t-2}+1}+n-s\left(k_{t}\right) .
$$

Similarly, if $k_{t-2}+2 \neq k_{t-1}$ then

$$
a_{k_{t}+2}=a_{k_{t-2}+2}+n-s\left(k_{t}\right) \text {, }
$$

and if $k_{t-2}+2=k_{t-1}$ then

$$
a_{k_{t}+2}=a_{k_{t-1}+1}+n-s\left(k_{t}\right) \text {. }
$$

Inequalities (24) and (25) imply

$$
s\left(k_{t}\right)+a_{k_{t}+2}-a_{k_{t}}>n,
$$

and as, by Lemma $9, s\left(k_{t}\right)<n+\sqrt{2 n} / 8$ and $a_{k_{t}}>\sqrt{2 n} / 4$ inequality (26) implies that

$$
a_{k_{t}+1}>a_{k_{t}+2}>a_{k_{t}}+n-n-\sqrt{2 n} / 8>\sqrt{2 n} / 8 .
$$

Let us partition $A$ by setting $A_{1}=L \cup\left\{a_{k_{t}+1}, a_{k_{t}+2}\right\}-\left\{a_{k_{t}}\right\}$ and $A_{2}=$ $A-A_{1}$. Then, by (26) and (27), we have $\sigma\left(A_{1}\right)>n+\sqrt{2 n} / 8$, so $\sigma\left(A_{2}\right)<n$ and thus $n \in \Sigma\left(A_{1}\right)$. Let $Q$ be a subset of $A_{1}$ such that $n=\sigma(Q)$. Since $s\left(k_{t}\right)-a_{k_{t}}<0$, inequality (26) implies that there is an $a_{j} \in L-\left\{a_{k_{t}}\right\}$ such that $Q=A_{1}-\left\{a_{j}\right\}$. Therefore, by (23),

$$
\begin{aligned}
n & =\sigma(Q)=\sigma\left(A_{1}\right)-a_{j}=\sigma(L)+a_{k_{t}+1}+a_{k_{t}+2}-a_{k_{t}}-a_{j} \\
& =s\left(k_{t}\right)+a_{k_{t}+1}+a_{k_{t}+2}-a_{k_{t}}-a_{j} \\
& =s\left(k_{t}\right)+a_{k_{t-2}+1}+n-s\left(k_{t}\right)+a_{k_{t}+2}-a_{k_{t}}-a_{j} \\
& =n+\left(a_{k_{t-2}+1}-a_{j}+a_{k_{t}+2}-a_{k_{t}}\right) .
\end{aligned}
$$


However, we claim that

$$
a_{k_{t-2}+1}-a_{j}+a_{k_{t}+2}-a_{k_{t}} \neq 0 .
$$

It is trivial that (28) holds for $a_{j}=a_{k_{t-2}+1}$. If $a_{j}=a_{k_{t-2}+2}$, then $k_{t-2}+2 \neq$ $k_{t-1}$, so, by (23) and (24), $a_{k_{t-2}+1}-a_{j}+a_{k_{t}+2}-a_{k_{t}}=a_{k_{t-2}+1}+n-s\left(k_{t}\right)-$ $a_{k_{t}}>a_{k_{t-2}+1}+n-s\left(k_{t}\right)-a_{k_{t}+1}=0$. If $a_{j}=a_{k_{t-1}+1}$, then, by (26) and (27), $a_{k_{t-2}+1}+a_{k_{t}+2}>a_{k_{t-1}}+\sqrt{2 n} / 8>a_{j}+a_{k_{t}}$, so, inequality (28) holds again. In fact, these are all the cases since $k_{t-1}-k_{t-2} \leq 3$ and $k_{t}-k_{t-1}=2$. Thus (28) does hold, which is a contradiction.

(ii) Assume now that $a_{k_{t}+b-1}=a_{k_{t}}+n-s\left(k_{t}\right)$. We shall show that $h=k_{t}+b$ will do for the claim in the lemma. Clearly

$$
\sigma\left(L \cup\left\{a_{h}\right\}\right)-a_{k_{t}}=s\left(k_{t}\right)+a_{k_{t}+b}-a_{k_{t}}<s\left(k_{t}\right)+a_{k_{t}+b-1}-a_{k_{t}}=n .
$$

As $\sigma(L)>n$ and $a_{k_{t}}$ is the smallest term in $L$, this implies $n \notin \Sigma(L \cup$ $\left\{a_{k_{t}+b}\right\}$ ), showing the first assertion of the lemma. To see the second assertion, note that

$$
\begin{aligned}
& \sigma(K)+\sum_{i=k_{t}+1}^{h-1} a_{i}<s\left(k_{t}\right)-\sum_{i=k_{t-2}+1}^{k_{t-1}-2} a_{i}-\sum_{i=k_{t-1}+1}^{k_{t}} a_{i}+\sum_{i=k_{t}+1}^{k_{t}+b-1} a_{i} \\
&= s\left(k_{t}\right)-\left(\sum_{i=k_{t-2}+1}^{k_{t-1}-2} a_{i}+\sum_{i=k_{t-1}+1}^{k_{t}-1} a_{i}-\sum_{i=k_{t}+1}^{k_{t}+b-2} a_{i}\right) \\
&-a_{k_{t}}+a_{k_{t}+b-1} \\
&< s\left(k_{t}\right)-a_{k_{t}}+a_{k_{t}+b-1}=n,
\end{aligned}
$$

since the sum in parentheses is positive as there are $b-2$ positive terms and $b-2$ smaller negative terms. This completes the proof of the lemma.

Armed with Lemma 12, the proof of Theorem 6 is easily completed. Let $h$ be the index whose existence is guaranteed by Lemma 12 .

Assume first that $a_{h} \geq \sqrt{2 n} / 8$. Let $A_{1}=L \cup\left\{a_{h}\right\}$ and $A_{2}=A-A_{1}=$ $K \cup\left\{a_{k_{t}+1}, \ldots, a_{m}\right\}-\left\{a_{h}\right\}$. Then $\sigma\left(A_{1}\right)>n+\sqrt{2 n} / 8$, so $\sigma\left(A_{2}\right)<n$, and so $n \notin \Sigma\left(A_{2}\right)$. However, by Lemma $11, n \notin \Sigma\left(A_{1}\right)$. This contradicts our assumption on the set $A$.

Let us assume then that $a_{h}<\sqrt{2 n} / 8$. Then $\sum_{i=h}^{m} a_{i} \geq \sqrt{2 n} / 8$. Indeed, otherwise let $A_{1}=L \cup\left\{a_{h}, \ldots, a_{m}\right\}$ and $A_{2}=A-A_{1}=K \cup$ $\left\{a_{k_{t}+1}, \ldots, a_{h-1}\right\}$. Lemmas 10 and 11 imply that $n \notin \Sigma\left(A_{1}\right)$. However, by Lemma 12 ,

$$
\sigma\left(A_{2}\right)=\sigma(K)+\sum_{i=k_{t}+1}^{h-1} a_{i}<n,
$$


so $n \notin \Sigma\left(A_{2}\right)$, contradicting our assumption. Therefore $\sum_{i=h}^{m} a_{i} \geq \sqrt{2 n} / 8$, as claimed.

Since $a_{h}<\sqrt{2 n} / 8,0<s\left(k_{t}\right)-n<\sqrt{2 n} / 8$ and $a_{h}>a_{h+1}>\ldots>a_{m}$, there exists an index $l, h \leq l \leq m$, such that

$$
n+\sqrt{2 n} / 8-s\left(k_{t}\right) \leq \sum_{i=h}^{l} a_{i}<n+\sqrt{2 n} / 4-s\left(k_{t}\right) \leq n+a_{k_{t}}-s\left(k_{t}\right) .
$$

Let $Q=\left\{a_{h}, a_{h+1}, \ldots, a_{l}\right\}$ so that $\sigma(Q)<n+a_{k_{t}}-s\left(k_{t}\right)$. Set $A_{1}=L \cup Q$ and

$$
A_{2}=A-A_{1}=K \cup\left\{a_{k_{t}+1}, \ldots, a_{h-1}\right\} \cup\left\{a_{l+1}, \ldots, a_{m}\right\} .
$$

Then, by Lemma $10, n \notin \Sigma\left(A_{1}\right)$. However, by the definition of $l$,

$$
\sigma\left(A_{1}\right)=s\left(k_{t}\right)+\sum_{i=h}^{l} a_{i} \geq n+\sqrt{2 n} / 8,
$$

so $\sigma\left(A_{2}\right)<n$ and hence $n \notin \Sigma\left(A_{2}\right)$, contradicting our assumption on $A$ and completing the proof of the theorem.

It is tempting to conjecture that $g_{2}(n)=f_{2}(n)\left(f_{2}(n)+1\right) / 2$ but, if true, this seems to be rather difficult. It may be easier to show that, as we suspect, $\left(g_{2}(n)-2 n\right) / \sqrt{n} \rightarrow \infty$.

\section{References}

[1] S. A. Burr and P. Erdős, A Ramsey-type property in additive number theory, Glasgow Math. J. 27 (1985), 5-10.

[2] P. Erdös and H. Heilbronn, On the addition of residue classes $\bmod p$, Acta Arith 9 (1964), 149-159.

[3] R. L. Graham, Rudiments of Ramsey Theory, CBMS Regional Conf. Ser. in Math. 45, Amer. Math. Soc., 1981.

[4] R. L. Graham, B. L. Rothschild and J. H. Spencer, Ramsey Theory, 2nd ed. Wiley-Interscience, New York 1990.

[5] J. E. Olson, An addition theorem modulo p, J. Combin. Theory 5 (1968), 45-52.

DEPARTMENT OF PURE MATHEMATICS

AND MATHEMATICAL STATISTICS UNIVERSITY OF CAMBRIDGE

16 MILL LANE

CAMBRIDGE CB2 1SB, ENGLAND
MATHEMATICAL INSTITUTE HUNGARIAN ACADEMY OF SCIENCES P.O. BOX 127 H-1364 BUDAPEST, HUNGARY 